\title{
Embedded Multiplexed Polymer Optical Fiber Sensor for Esophageal Manometry
}

\author{
Bram Van Hoe, Erwin Bosman, \\ Jeroen Missinne, Geert Van \\ Steenberge and Peter Van Daele \\ Centre for Microsystems Technology \\ ELIS/INTEC Dept., \\ Ghent University-IMEC \\ Technologiepark 914A \\ 9052 Zwijnaarde, Belgium \\ Email: bram.vanhoe@elis.ugent.be
}

\author{
Wei Zhang, Ian Johnson, \\ Kate Sugden and David J. Webb \\ Photonics Research Group \\ Aston University \\ Birmingham B4 7ET, UK \\ Email: d.j.webb@aston.ac.uk
}

\author{
Kyriacos Kalli \\ Dept. of Electrical Engineering \\ and Information Technology \\ Cyprus University of Technology \\ Lemessos 3036, Cyprus \\ Email: kyriacos.kalli@cut.ac.cy
}

\begin{abstract}
There is a growing interest for esophageal measurements which can provide important and reliable data when diagnosing the motor function of the sphincters and the esophageal body. Biocompatibility, sensing resolution and the comfort of the patient are key parameters for manometric sensing systems. A new sensing approach which could fulfill all these needs is presented in this paper consisting of an embedded polymer fiber sensor, based on multiplexed fiber Bragg gratings. A response to a radial pressure almost 6 times that of a comparable silica fiber based sensor is obtained.
\end{abstract}

\section{INTRODUCTION}

Manometry measures pressure within the esophageal lumen and sphincters and is performed to investigate the cause of functional dysphagia, unexplained non-cardiac chest pain, and in the pre-operative work up of patients referred for antireflux surgery [1]. There is an increasing demand for patient-friendly, highly sensitive and biocompatible pressure sensors, embedded in cylindrical tubes, which can easily be multiplexed. The duration of the measurement, the mechanical flexibility of the sensor and the amount of pressure sensing points integrated in one sensing tube are key parameters for esophageal sensors.

Traditional systems include sensors based on solid-state catheters or MEMS technology [2]. Recently, also fiber optic alternatives are looked into providing multiplexing possibilities and fast real-time measurements [3].

The paper we present is based on the latter sensing mechanism: a fiber with multiple Bragg gratings is embedded in a cylindrical tube. Unlike other systems based on Bragg gratings, we are using polymer optical fibers (POF) instead of silica resulting in improved pressure sensitivity and inherent biocompatibility [4]. Moreover, the tube embedding host material is a flexible and stretchable polymer providing excellent compatibility with the sensor device and enhancing the patient's comfort when the esophageal investigation is performed. Embedding technology to integrate both silica and polymer fiber sensors in planar stretchable host materials has been reported earlier [5] and is now extended to cylindrical tubes. The polymer embedding material is acting as a transducer; its properties define the sensitivity of the tube.

\section{SENSOR PRINCIPLE}

A fiber Bragg grating (FBG) sensor takes the form of a permanent periodic spatial modulation of the refractive index along the core of an optical fiber, commonly induced using ultraviolet light. Under the influence of temperature or strain, both the period of the modulation and the mean index of the core mode change leading to a shift in the wavelength reflected back down the fiber by this diffractive structure. Over the last 20 years of intensive development silica fiber Bragg grating technology has grown increasingly mature and is now commercially exploited in a range of applications such as oil and gas exploration [6].

In contrast, polymer optical fiber Bragg grating (POFBG) sensors date back only a little over 10 years [7] and represent a much less mature technology. POFBGs have some disadvantages compared to silica technology: the fiber is more lossy, especially in the near infra-red, and single or fewmoded optical fiber is not currently widely available. Nevertheless, polymers possess some interesting material properties that suggest advantages over silica in certain situations. In this work we seek to exploit two features. Firstly, POF has an elastic modulus roughly 25 times smaller than silica; consequently, although the strain sensitivity of POFBGs is only marginally higher than their silica counterparts, the stress sensitivity is more than an order of magnitude higher. Secondly, for in vivo applications, when 
using glass fibers great care must be taken to guard against a fiber break which could exhibit a serious sharp hazard.

The sensor concept is shown in Figure 1. The interrogation system has been designed to work with a multimode fiber grating and comprises of an Exalos broadband SLED light source operating around $850 \mathrm{~nm}$ [8]. The light passes through a 1x2 multimode coupler so that the reflected signal from the sensor tube can be measured using an Ocean Optics HR2000+ spectrometer. The POFBG is embedded in a $4.65 \mathrm{~mm}$ diameter compliant cylinder ultimately designed to pass into the esophagus through the nasal cavity. The sensor is designed to measure the lateral squeezing provided by the swallowing process. It is important to stress that we are not dealing with an isotropic pressure but rather a radial squeezing which causes an elongation of the cylinder as a result of Poisson's ratio.

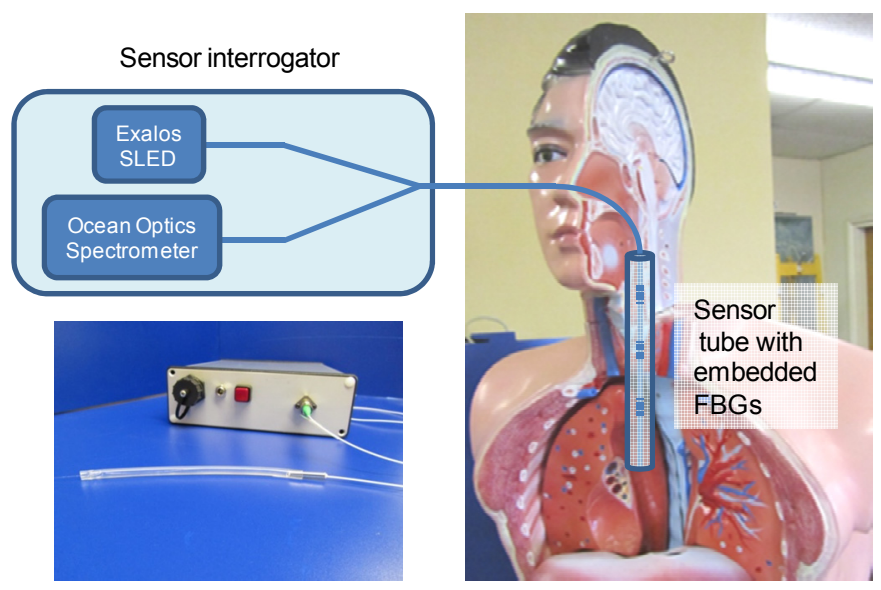

Figure 1: Sensor concept with the actual system bottom left.

\section{FABRICATION METHODS}

\section{A. Sensor fabrication}

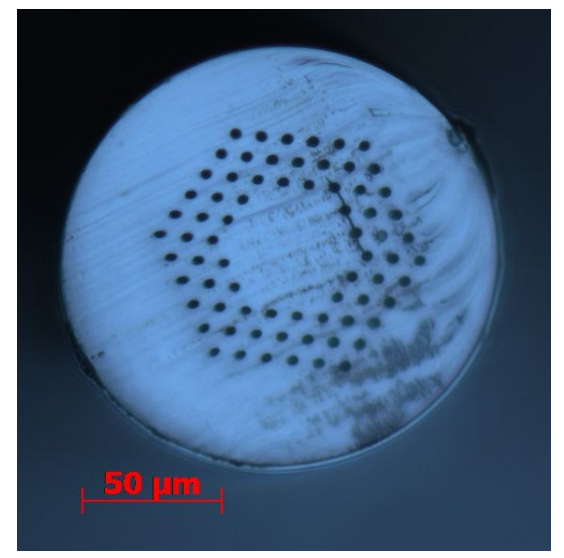

Figure 2: Microscope image of microstructured POF.

The POF used in this work was obtained from Kyriama (Australia) and is a microstructured fiber with a 50 micron core surrounded by three rings of holes, see Figure 2. The fiber was manufactured from nominally pure poly(methyl methacrylate). POFBGs can be recorded in such fiber using
$325 \mathrm{~nm}$ light from a HeCd laser [9]. For this work either two or three gratings were recorded in a $20 \mathrm{~cm}$ length, separated by $2 \mathrm{~cm}$. The gratings had a length of approximately $2 \mathrm{~mm}$ and were fabricated by exposing the fiber for approximately 50 minutes to the $30 \mathrm{~mW}$ laser beam after it had passed through a phase mask. In the case of the two grating sensor, two masks were used, designed to produce gratings at $861 \mathrm{~nm}$ and $827 \mathrm{~nm}$. For the three grating device, a grating was recorded at $861 \mathrm{~nm}$ and then the fiber was annealed at $70^{\circ} \mathrm{C}$ for 24 hours which reduced the Bragg wavelength by $16 \mathrm{~nm}$ [10]. Following the procedure, a second and third grating were recorded as described above. The end of the POF was glued to a $50 \mu \mathrm{m}$ step index silica pigtail, with the joint protected using a metallic tube filled with silicone. To allow comparison between the two fiber types, a single FBG was recorded in silica fiber (SMF) at a Bragg wavelength of 1549nm.

\section{B. Sensor embedding}

The tubular embedding process of a multiplexed polymer fiber sensor is schematically shown in Figure 3. The metal tube, protecting the glue joint, perfectly fits in the sensing tube. Dow Corning [11] Silastic ${ }^{\circledR}$ Medical Grade Tubing (outer diameter of $4.65 \mathrm{~mm}$ and inner diameter of $3.35 \mathrm{~mm}$ ) is used as a cylindrical tube and Sylgard $^{\circledR}$ 184, a Polydimethylsiloxane material from Dow Corning (PDMS), is used as a flexible and stretchable embedding material. The tube is sealed on one end with a circular PMMA barrier accommodated with an injection inlet and a fiber outlet. This host material is injected in a liquid state via the injection inlet. By using a fiber clamp on a microstage, one can adjust the position of the fiber in the tube and prestrain the fiber before the final curing process is initiated. This way the fiber can be mounted in the middle of the tube and the position of the Bragg gratings can be accurately controlled.



Figure 3: Schematic view of the fiber embedding set-up.

Figure 4 (a) is depicting a flexible polymer sensing tube and the PMMA sealing is shown in Figure 4 (b). When illuminating the sensing tube with a $635 \mathrm{~nm}$ light source, the position of the gratings can be seen within the tube (shown in Figure 5). Similar tubes with silica fiber sensors are fabricated as a reference for characterization measurements. An example of such a tube is shown in Figure 6.

Spectral analysis was performed before and after embedding the fiber sensors in the tubes. The resulting characteristics are shown in Figure 7 and Figure 8. Curing of the PDMS material is performed at $60^{\circ} \mathrm{C}$ for the silica fiber and at room temperature for the polymer fiber in order to 
avoid further annealing of the Bragg gratings. The blue wavelength shift of the silica grating is caused by the coefficient of thermal expansion mismatch between the PDMS $\left(\sim 300 \mathrm{ppm} /{ }^{\circ} \mathrm{C}\right)$ and silica $\left(\sim 0.55 \mathrm{ppm} /{ }^{\circ} \mathrm{C}\right)$. The red wavelength shift of the polymer gratings is due to a prestraining step after the PDMS injecting to have the fiber positioned as straight as possible.



(a)

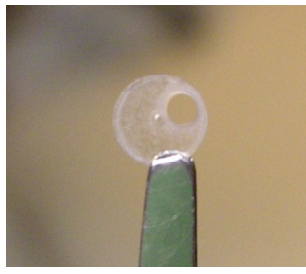

(b)
Figure 4: (a) Flexible polymer fiber sensing tube. (b) PMMA sealing plate with fiber and injection inlet.

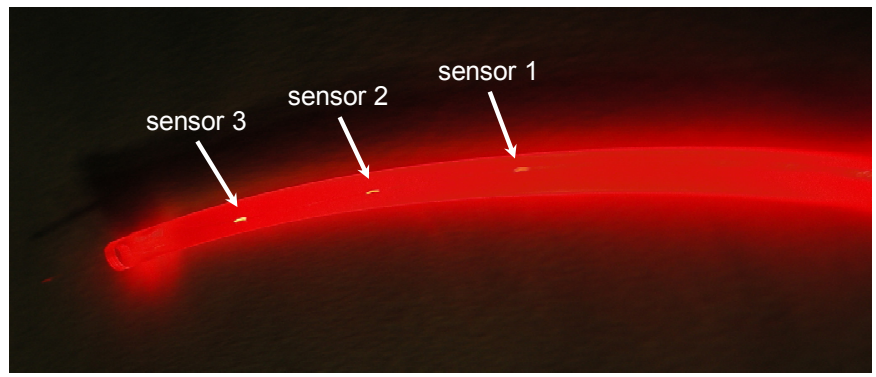

Figure 5: Multiplexed embedded polymer fiber sensing tube illuminated with a $635 \mathrm{~nm}$ light source.

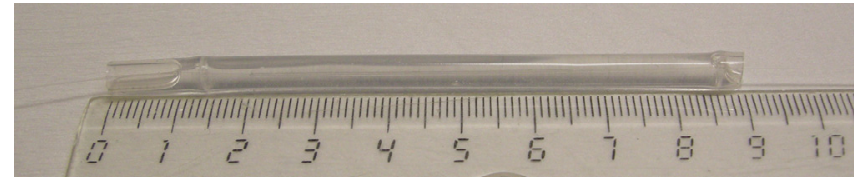

Figure 6: Silica fiber sensing tube.

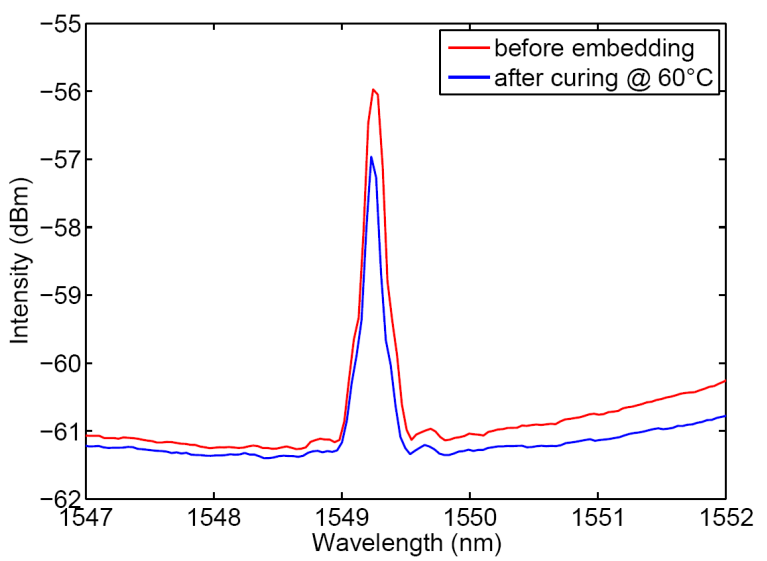

Figure 7: Spectral response of a silica fiber sensing tube, before and after embedding.

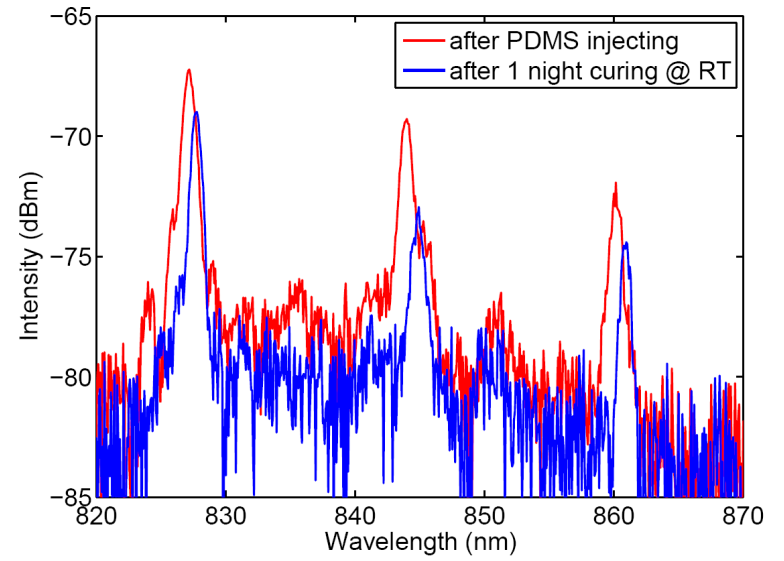

Figure 8: Spectral response of a multiplexed polymer fiber sensing tube, before and after PDMS polymerization.

\section{CHARACTERIZATION}

To quantify the sensor performance, two compliant tubes were placed on a metal plate. One of the tubes is based on the embedded silica SMF grating of $1549 \mathrm{~nm}$ while the other is embedded with an array of two POFBGs of $827 \mathrm{~nm}$ and $861 \mathrm{~nm}$. A metal beam with a width of $10 \mathrm{~mm}$ was placed on these two tubes. The cylindrical tubes were carefully aligned so the silica FBG and one of the POFBGs were positioned precisely under the beam for test. Weights were gradually applied onto the beam to simulate the radial squeezing, as shown in Figure 9. Since both tubes have the same diameter the pressure can be equally applied to the gratings under test.

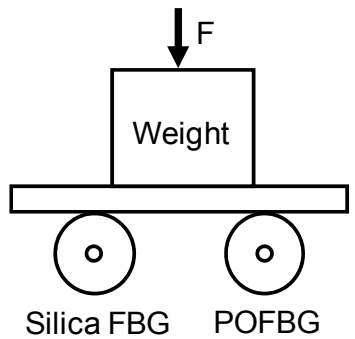

Figure 9: Pressure characterization arrangement.

As the weights were gradually applied the transversal force caused an elongation of the cylinder as a result of Poisson's ratio. The Bragg wavelengths of the gratings vary with the applied transversal force. Two broadband light sources centered at $850 \mathrm{~nm}$ and $1550 \mathrm{~nm}$ were used for POFBG and silica FBG, respectively. The Ibsen I-MON 850 wavelength interrogator was used to monitor the wavelength change of the POFBG while an I-MON 400 was used for the silica FBG.

\section{RESULTS AND DISCUSSION}

Figure 10 shows the response of a POFBG and the silica FBG to small loads. It may be seen that the wavelength shifts obtained are much larger in the case of the POFBG, however there is more noise visible in the data from this device. 


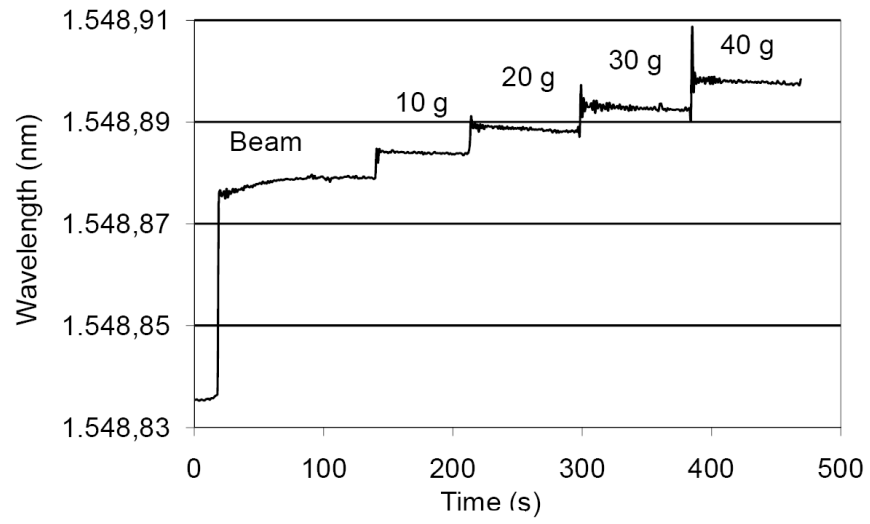

(a)

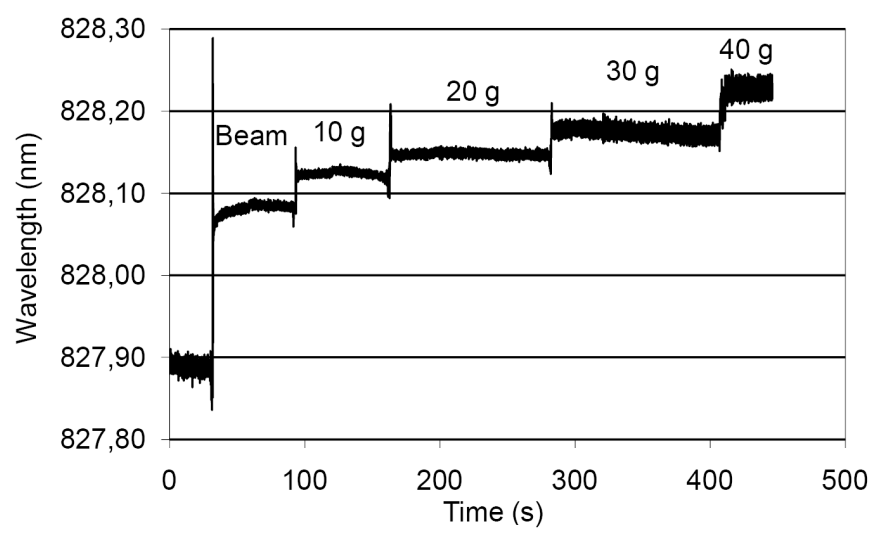

(b)

Figure 10: Response of silica (a) and POF (b) sensors to small forces on the beam.

The response of the device was also tested with larger forces, see Figure 11. It can be seen that the response of the POFBG is a factor of $0.838 / 0.276=3.04$ times greater than the silica device. It should be noted though that because of the different wavelengths of the two devices, the same strain would produce a wavelength shift in the silica device larger than that in the POF by the ratio of the wavelengths, namely 1.87. Consequently in terms of normalized sensitivity, the POF device performs a factor of $3.04 \times 1.87=5.68$ better than that based on silica fiber.

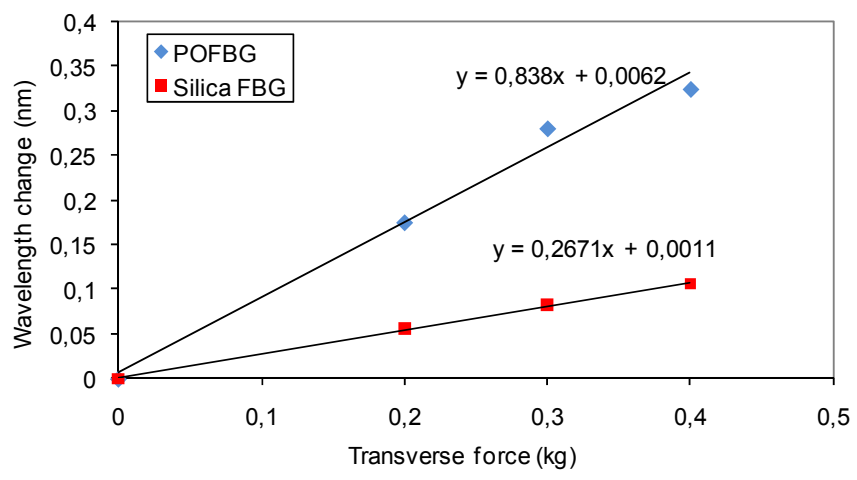

Figure 11: Response of silica and POF sensors to larger forces on the beam.
Typical esophageal calibration measurements are performed with applied loads between 0 and 200g [12]. End user requirements typically include a pressure range of $300 \mathrm{mmHg}$ and a resolution of $1 \mathrm{mmHg}$. Also the amount of multiplexed sensors has to be further increased to 10 sensors separated by a typical distance of $2 \mathrm{~cm}$.

\section{CONCLUSION}

We have reported on initial proof of principle tests performed on an esophageal pressure monitor. The use of POF rather than silica fiber enhances the sensitivity by a factor of 5.68 while reducing the potential risk from fiber breakage. The POF sensor investigated displays a higher level of noise than the silica device; we believe this is due to a nonequilibrium mode distribution in the multimode fiber sensor. Experimental work is ongoing to mitigate this problem.

\section{ACKNOWLEDGMENT}

This work is partially conducted in the framework of the project Phosfos, Photonic Skins for Optical Sensing (funded within the EU-FP7 program) [13]. The research of B. Van Hoe is supported by a Ph.D grant of the Institute for the Promotion of Innovation through Science and Technology in Flanders (IWT-Vlaanderen).

\section{REFERENCES}

[1] M. R. Fox, A. J. Bredenoord, "Oesophageal high-resolution manometry: moving from research into clinical practice", Gut 57, 405423, 2008

[2] Y. Haga, M. Esashi, "Biomedical Microsystems for Minimally Invasive Diagnosis and Treatment", Proceedings of the IEEE, vol. 92, nr. 1, 2004.

[3] S. Voigt, J. Mehner, M. Rothharadt, M. Becker, T. Lüpke., C. Thieroff, A. Teubner, "Homogeneous catheter for esophagus high-resolution manometry using fiber Bragg gratings", Proceedings of the SPIE, vol. 7559B, 75590, 2010.

[4] K. Kalli and D. J. Webb, "Polymer optical fiber based sensors", in Advanced Fiber Optics, L. Thevenaz, EPFL Press, 2011.

[5] B. Van Hoe, G. Van Steenberge, E. Bosman, J. Missinne, P. Van Daele, T. Geernaert et al., "Optical fiber sensors embedded in flexible polymer foils", Proceedings of the SPIE, vol. 7726, 772603, 2010.

[6] Advanced Fiber Optics, L. Thevenaz, EPFL Press, 2011

[7] Z. Xiong, G.D. Peng, B. Wu, P.L. Chu, "Highly tunable Bragg gratings in single-mode polymer optical fibers", IEEE Photon. Technol. Lett., vol. 11, nr. 3, pp. 352-354, 1999.

[8] Exalos, http://www.exalos.com.

[9] H. Dobb, D.J. Webb, K. Kalli, A. Argyros, M.C.J. Large, M.A. van Eijkelenborg, "Continuous wave ultraviolet light-induced fiber Bragg gratings in few- and single-mode microstructured polymer optical fibers", Opt. Lett., vol. 30, nr. 24, pp. 3296-3298, 2005.

[10] I.P. Johnson, D.J. Webb, and K. Kalli, "Utilisation of thermal annealing to record multiplexed FBG sensors in multimode microstructured polymer optical fibre", Proceedings of the SPIE, vol. 7753, 77536T, 2011.

[11] Dow Corning, http://www.dowcorning.com.

[12] C. O. H. Russell, N. Bright, G. Buthpitiya, L. Alexander, C. Walton, G. Whelan, "Oesophageal propulsive force and its relation to manometric pressure", Gut, 33, 727-732, 1992.

[13] PHOSFOS, Photonic Skins for Optical Sensing, http://www.phosfos.eu. 